\title{
The LXXLL motif of murine forkhead transcription factor FoxO1 mediates Sirt1-dependent transcriptional activity
}

\author{
Jun Nakae, ${ }^{1}$ Yongheng Cao, ${ }^{1}$ Hiroaki Daitoku, ${ }^{2}$ Akiyoshi Fukamizu, ${ }^{2}$ Wataru Ogawa, ${ }^{1}$ \\ Yoshihiko Yano, ${ }^{1}$ and Yoshitake Hayashi ${ }^{3}$
}

\begin{abstract}
1Department of Clinical Molecular Medicine, Division of Diabetes, Digestive and Kidney Disease, Kobe University Graduate School of Medicine, Kobe, Japan. ${ }^{2}$ Center for Tsukuba Advanced Research Alliance, University of Tsukuba, Tsukuba, Japan. ${ }^{3}$ Division of Molecular Medicine and Medical Genetics, International Center for Medical Research and Treatment (ICMRT), Kobe University Graduate School of Medicine, Kobe, Japan.
\end{abstract}

\begin{abstract}
The forkhead transcription factor FoxO1 has been identified as a negative regulator of insulin/IGF-1 signaling. Its function is inhibited by phosphorylation and nuclear exclusion through a PI3K-dependent pathway. However, the structure/function relationship of FoxO1 has not been elucidated completely. In this study, we carried out mutation analysis of the FoxO1 coactivator-interacting LXXLL motif (amino acids 459-463). Expression of a 3A/LXXAA mutant, in which 3 Akt phosphorylation sites (T24, S253, and S316) and 2 leucine residues in the LXXLL motif (L462 and L463) were replaced by alanine, decreased both Igfbp-1 and G6Pase promoter activity and endogenous $I g f b p-1$ and G6Pase gene expression in simian virus 40-transformed (SV40-transformed) hepatocytes. Importantly, mutagenesis of the LXXLL motif eliminated FoxO1 interaction with the nicotinamide adenine dinucleotide-dependent (NAD-dependent) deacetylase sirtuin 1 (Sirt1), sustained the acetylated state of FoxO1, and made FoxO1 nicotinamide and resveratrol insensitive, supporting a role for this motif in Sirt1 binding. Furthermore, intravenous administration of adenovirus encoding 3A/LXXAA FoxO1 into $L e \mathrm{pr}^{d b / d b}$ mice decreased fasting blood glucose levels and improved glucose tolerance and was accompanied by reduced G6Pase and Igfbp-1 gene expression and increased hepatic glycogen content. In conclusion, the LXXLL motif of FoxO1 may have an important role for its transcriptional activity and Sirt1 binding and should be a target site for regulation of gene expression of FoxO1 target genes and glucose metabolism in vivo.
\end{abstract}

\section{Introduction}

Members of the forkhead box O (FoxO) family of transcription factors, including FoxO1 (previously described as FKHR), FoxO3a (FKHRL1), FoxO4 (AFX), and FoxO6, are phosphorylated by insulin/IGF-1 through a PI3K/Akt-dependent pathway and excluded from nucleus to inhibit their transcriptional activity $(1$, $2)$. FoxOs cause cell cycle arrest through induction of $p 27, p 21$, cyclin $B$, polo-like kinase, the $\mathrm{Rb}$ family-related protein $\mathrm{p} 130$, and cyclin G2 (3-8), apoptosis through induction of Fas ligand and Bim $(9,10)$, DNA repair through GADD45 $(11,12)$, stress resistance through $M n S O D$ (13-15), and regulation of glucose and lipid metabolism through induction of G6Pase, apoC-III, and Igfbp-1 expression (16-18). Studies using genetically modified mice, such as knockout and transgenic mice, indicate that each FoxO family member has a different role in vivo. FoxO1 knockout is embryonic lethal due to defective angiogenesis (19). Haploinsufficiency of FoxO1 protects against insulin resistance, and overexpression of a constitutively active FoxO1 mutant in liver and/or pancreatic $\beta$ cells (Ttr S253A) causes insulin resistance (17). On the other hand, ablation of FoxO3a causes premature ovarian failure as a result of accelerated differentiation and consequent depletion of primary ovarian follicles $(19,20)$.

Nonstandard abbreviations used: ADA, T24A/S253D/S316A; 8-Br-cAMP, 8-bromoadenosine cAMP; Cbp, CREB-binding protein; ChIP, chromatin IP; FoxO, forkhead box O; HA, hemagglutinin; HDAC, histone deacetylase; IBMX, 3-isobutyl1-methylxanthine; NAD, nicotinamide adenine dinucleotide; Sir2, silent information regulator 2; Sirt1, sirtuin 1; SV40, simian virus 40 .

Conflict of interest: The authors have declared no conflict of interest exists. Citation for this article: J. Clin. Invest. 116:2473-2483 (2006). doi:10.1172/JCI25518.
FoxO family members have a transactivation domain in their $\mathrm{C}$ terminal region. Control of gene expression is a complex process that involves assembly of multiple transcription factors at the distal enhancer region and basal transcriptional machinery at the core promoter region of target genes (21). Gene expression involves the sequential assembly of an array of coregulatory proteins that include coactivators. Coactivator complexes are recruited via specific protein-protein interactions, often mediated by specific interaction motifs in the transactivation domains of transcription factors (22). The LXXLL motif (where L is a leucine and X any amino acid) was originally identified in a variety of coactivators, such as p300/CREB-binding protein (p300/Cbp) and RIP-140. It is described as a signature motif that mediates the recruitment of coactivators by the nuclear hormone receptors $(23,24)$. Specific LXXLL motifs of nuclear receptor coactivators (NcoAs) mediate ligand-dependent interaction with nuclear receptors as well as p300/Cbp (23). Thus, LXXLL motifs have evolved to serve overlapping roles that are likely to permit both receptor-specific and ligand-specific assembly of a coactivator complex, and these recognition motifs underlie the recruitment of coactivator complexes required for nuclear receptor function.

In the present study, we identify an LXXLL motif from amino acids 459-463 in murine FoxO1 and demonstrate that the LXXLL motif is quite important for full activation of transcription by FoxO 1 in Igfbp-1 or G6Pase promoter assay and endogenous I $g f b p-1$ and G6Pase gene expression in simian virus 40-transformed (SV40transformed) hepatocytes. Furthermore, we demonstrate that the LXXLL motif has a critical role in the interaction of FoxO1 with sirtuin 1 (Sirt1) and that the transcriptional activity of FoxO1 is 
Table 1

Sequence alignment of LXXLL motifs in members of FoxO family

\section{Proteins}

Human F0X01

Human FOX03A

Human FOXO4

Murine Fox01

Murine Fox03a

Murine Fox04

$X$. laevis Fox01

$X$. laevis $\mathrm{F} 0 \mathrm{x} 03$

C. elegans DAF- 16

D. melanogaster $\mathrm{dFOXO}$
Amino acid sequences

MSQYNCAPGLLKELLTSDSPPHNDIM SSMSHYGNQTLQDLLTSDSLSHSDV GEGCFSSSQALEALLTSDTPPPPAD LNQYNNAPGLLKELLTSDSPPHNDIM SSVSHYGNQTLQDLLASDSLSHSDV GEGCFSSSQSLEALLTSDTPPPPAD VGQYPVPAGLLKELLTSDSPPHNDIL SSMNHYSNQSLQDLLNTDTLSHSDVL AQHTVASSSALPIDLENLTLPDQPL NTLQPQSQCLLHRSLNCSCMHNARD

dependent on the LXXLL motif and interaction with Sirt1. Finally, we show that acute overexpression of a mutant FoxO1 in which all 3 Akt phosphorylation sites (threonine 24, serine 253, and serine 316) and 2 leucine residues at amino acids 462 and 463 were replaced by alanine ameliorates insulin resistance in Lepr ${ }^{d b / d b}$ mice. We suggest the possibility that the LXXLL motif of FoxO1 should be a target site for regulation of glucose metabolism in vivo.

\section{Results}

The LXXLL motif is conserved among FoxO family members. We identified an LXXLL motif at amino acids 459-463 in murine FoxO1, which is highly conserved among FoxO family members in human, murine,

and Xenopus laevis and weakly conserved in Drosophila melanogaster and Caenorhabditis elegans (Table 1). This finding suggests that the LXXLL motif may have an important role in the function of FoxO proteins.

Mutagenesis of the LXXLL motif of FoxO1 abolishes Igfbp-1 and G6Pase promoter activity. At first, in order to investigate roles of the LXXLL motif of FoxO1, we constructed an LXXAA mutant in which leucine 462 and 463 were substituted by alanine (Figure 1A) and performed Igfbp-1 and G6Pase promoter assays using this mutant FoxO1 in SV40-transformed hepatocytes (25). Both Igfbp-1 and G6Pase have already been identified as target genes of FoxO1 (16, $26)$. In the absence of serum, WT FoxO1 increased Igfbp-1 and G6Pase promoter activity by 2.5- and 3.2-fold compared with control, respectively (Figure 1, B and C). However, the LXXAA mutant increased Igfbp-1 and G6Pase promoter activity by only 1.3 - and 1.9-fold, respectively (Figure 1, and C). These data indicate that disruption of the LXXAA motif of FoxO1 decreases its transcriptional activity on its target genes in the absence of serum.

Transcriptional activity of FoxO1 is largely dependent on its subcellular localization (1). Although serum deprivation increases nuclear localization of FoxO1, the amount of FoxO1 that is localized in the nucleus is about $60 \%$ and cannot reach $100 \%$ in this cell line (data not shown). In order to investigate effects of disruption of the LXXAA motif on transcriptional regulation without considering subcellular localization of FoxO1, we constructed 3A/LXXAA mutant in which all Akt phosphorylation sites and leucines 462 and 463 were also substituted by alanine (Figure 1A). Immunofluorescence studies demonstrated that subcellular localization of the 3A/LXXAA mutant was the same as in the $3 \mathrm{~A}$

\section{Figure 1}

Scheme and subcellular localization of FoxO1 mutants in SV40-transformed hepatocytes and expression of an lgfbp-1/luciferase or G6Pase/luciferase reporter gene in SV40-transformed hepatocytes transfected with WT or LXXAA FoxO1. (A) Scheme of WT, LXXAA FoxO1, 3A FoxO1, and 3A/LXXAA FoxO1. (B) Expression of cMycWT (lane 2) and LXXAA FoxO1 (lane 3) in SV40-transformed hepatocytes was detected. (C) After overnight serum deprivation and induction with dexamethasone, 8-Br-cAMP, and IBMX in the presence or absence of insulin (100 $\mathrm{nM})$ as described in Methods, cells were harvested and luciferase activity was measured. *Statistically significant difference between control and WT-transfected cells, $P<0.001$ by 1 -way ANOVA; ** statistically significant difference between WT-and LXXAA-transfected cells, $P<0.005$ by 1 -way ANOVA. Data represent mean \pm SEM from 3 independent transfection experiments. Following transient transfection, hepatocytes were seeded onto 4-well slide culture chambers and incubated in various conditions, including serum deprivation, for 24 hours (D), stimulation with insulin (100 nM, 20 minutes) (E), incubation with serum $(\mathbf{F})$, and stimulation with $\mathrm{H}_{2} \mathrm{O}_{2}(250 \mu \mathrm{M}$, 4 hours) (G). Myc epitope-tagged FoxO1 was visualized with anti-cMyc monoclonal antibody and FITC-conjugated anti-mouse IgG.
A

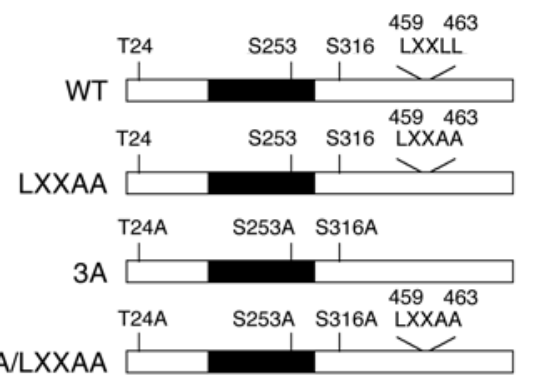

B

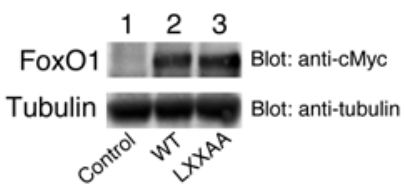

c
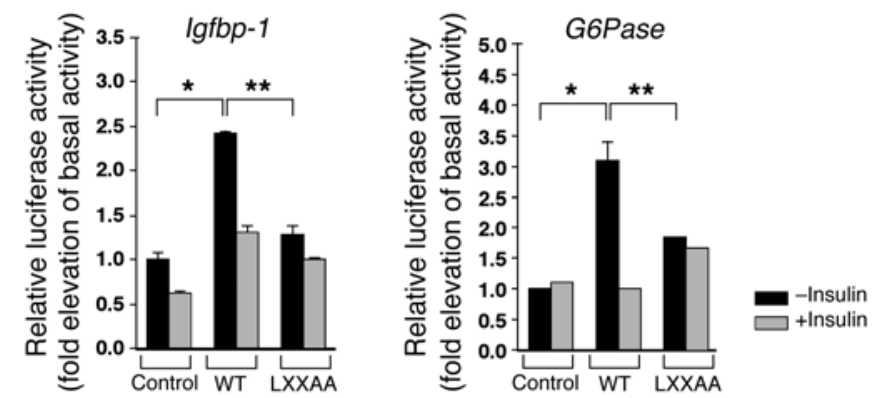

D Serum
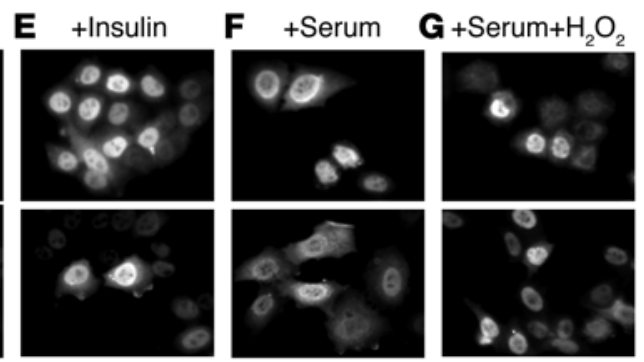


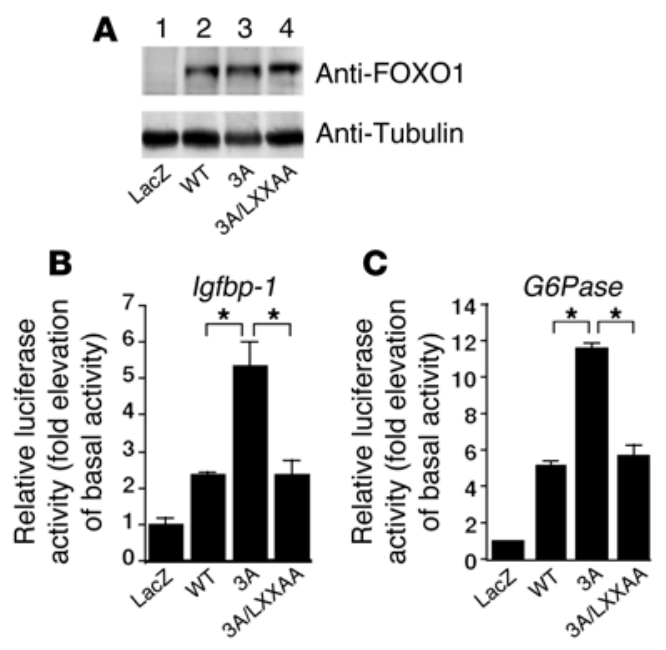

Figure 2

Expression of an /gfbp-1/luciferase or G6Pase/luciferase reporter gene in SV40-transformed hepatocytes transduced with adenovirus encoding LacZ, WT FoxO1, 3A FoxO1, or 3A/LXXAA FoxO1. (A) Expression of HA-WT FoxO1, Flag 3A FoxO1, or 3A/LXXAA FoxO1 in SV40-transformed hepatocytes was detected. Lane 1, hepatocytes transduced with adenovirus encoding LacZ; lane 2, with WT; lane 3, with $3 \mathrm{~A}$ FoxO1; lane 4, with 3A/LXXAA FoxO1 at 10 MOI. After hepatocytes were transfected with /gfbp-1/(p925GL3) (B) or G6Pase/(PicaGene/ human G6Pase promoter-luciferase) (C), cells were infected with indicated adenovirus. Synthetic Renilla luciferase reporter vector (phRLSV40) was used as an internal control of transfection efficiency. After overnight serum deprivation and induction with dexamethasone, 8-BrCAMP, and IBMX as described in Methods, cells were harvested and luciferase activity was measured. *Statistically significant difference between 3A FoxO1- and WT FoxO1- or 3A/LXXAA FoxO1-transduced cells, $P<0.001$ by 1 -way ANOVA. Data represent mean \pm SEM from 3 independent experiments.

mutant, in which only 3 Akt phosphorylation sites were substituted by alanine (Figure 1A) under several conditions, i.e., it is constitutively nuclear (Figure 1, D-G). Using these mutant FoxO1, we investigated effects of disruption of the LXXLL motif of FoxO1 on transcriptional regulation of FoxO1 target genes without considering subcellular localization. We performed Igfbp-1 and G6Pase promoter assays to investigate the effects of mutagenesis of the LXXLL motif on target gene expression. We used adenovirus vectors encoding LacZ, WT, 3A, and 3A/LXXAA in order to fix FoxO1 expression level (Figure 2A). WT FoxO1 and 3A FoxO1 increased Igfbp-1 promoter activity by 2.3 - and 5.4-fold compared with LacZ, respectively (Figure $2 \mathrm{~B}$ ). However, the $3 \mathrm{~A} / \mathrm{LXXAA}$ mutant increased $I g f b p-1$ promoter activity by only 2.8 -fold (Figure $2 \mathrm{~B}$ ). Therefore, disruption of the LXXLL motif of $3 \mathrm{~A}$ FoxO1 decreased Igfbp-1 promoter activity by $47 \%$. Furthermore, WT FoxO1 and $3 \mathrm{~A}$ FoxO1 increased G6Pase promoter activity by 5.0- and 11.6-fold, respectively (Figure 2C). However, the 3A/LXXAA mutant increased G6Pase promoter activity by only 5.7-fold (Figure 2C). Therefore, disruption of the LXXLL motif of 3A FoxO1 decreased G6Pase promoter activity by $50 \%$. These data suggest that disruption of the LXXLL motif of FoxO1 abolishes Igfbp-1 and G6Pase promoter activity and that the LXXLL motif of FoxO1 plays an important role in mediating the transcriptional activity of FoxO1.

Mutagenesis of the LXXLL motif of FoxO1 inbibits endogenous Igfbp-1 and G6Pase gene expression. In order to investigate effects of disrupt- ing the LXXLL motif of FoxO1 on endogenous gene expression, we transduced SV40-transformed hepatocytes with adenovirus vector encoding WT FoxO1, 3A FoxO1, or 3A/LXXAA FoxO1 (Figure 3A). In this cell line, Igfbp-1 and G6Pase are induced in a dexamethasone/8-Br-cAMP/IBMX-dependent manner (8-Br-cAMP, 8-bromoadenosine cAMP; IBMX, 3-isobutyl-1-methylxanthine). After serum deprivation, dexamethasone/8-Br-cAMP/IBMX increased endogenous $I g f b p-1$ gene expression by 43 -fold compared with basal condition (data not shown). Furthermore, transduction with 3A FoxO1 increased dexamethasone/8-Br-cAMP/IBMXinduced $I g f b p-1$ gene expression by 23 -fold compared with LacZtransduced conditions (Figure $3 \mathrm{~B}$ ). However, transduction with $3 \mathrm{~A} / \mathrm{LXXAA}$ FoxO1 increased Igfbp-1 gene expression by only 5.2-fold compared with LacZ-transduced conditions (Figure 3B). Furthermore, we investigated the effects of overexpression of mutant FoxO1 protein on endogenous G6Pase gene expression in this cell line. Transduction with $3 \mathrm{~A}$ FoxO1 increased dexamethasone/8-Br-cAMP/IBMX-induced endogenous G6Pase by 2.6-fold compared with LacZ-transduced condition. However, transduction with 3A/LXXAA FoxO1 increased by only 1.8-fold (Figure 3C). These data indicate that FoxO1 regulates endogenous Igfbp-1 and G6Pase gene expression in SV40-transformed hepatocytes in an LXXLL motif-dependent manner.

Because the $\mathrm{C}$ terminal region of FoxO1 is a transactivation domain, truncation of the terminus $(\Delta 256)$ abolishes its transcriptional activity. In order to determine how much transcriptional activity the LXXLL motif contributes to full transcriptional activity of the terminus of FoxO1, we compared endogenous Igfbp-1 gene expression levels in SV40-transformed hepatocytes infected with adenovirus encoding 3A FoxO1, 3A/LXXAA FoxO1, or $\triangle 256$ FoxO1. In the absence of dexamethasone/8-Br-cAMP/ IBMX, 3A FoxO1, 3A/LXXAA FoxO1, or $\Delta 256$ FoxO1 induced endogenous Igfbp-1 gene expression by 9.9-, 4.3-, or 1.4-fold, respectively (Figure 3D). In the presence of dexamethasone/8-Br-cAMP/ IBMX, transduction with 3A FoxO1, 3A/LXXAA FoxO1, or $\Delta 256$ FoxO1 induced gene expression by 98-, 30-, or 6.6-fold, respectively (Figure 3D). These data suggest that $70 \%$ of transcriptional activity of FoxO1 depends on the LXXLL motif.

Disruption of the LXXLL motif in constitutively active mutant FoxO1 confers dominant-negative effect onto endogenous Igfbp-1 gene expression. In order to investigate whether disruption of the LXXLL motif of FoxO1 confers dominant-negative effect onto endogenous FoxO1 target gene expression, we transduced SV40transformed hepatocytes with adenoviruses encoding hemagglutinin-T24A/S253D/S316A (HA-ADA), which has already been reported to be constitutively active FoxO1 (16), and Flag-3A/LXXAA FoxO1 (Figure 4A), and examined endogenous Igfbp-1 gene expression by real-time PCR. In this cell line, HA-ADA FoxO1 induced endogenous $I g f b p-1$ gene expression by 25 -fold compared with nontransduced cells in the presence of dexamethasone/8-Br-cAMP/ IBMX (Figure 4B). However, overexpression of 3A/LXXAA FoxO1 inhibited endogenous $I g f b p-1$ gene expression induced by overexpression of HA-ADA by $65 \%$ and $70 \%$ in a dose-dependent manner (Figure 4B). These data suggest that disruption of the LXXLL motif of FoxO1 confers dominant-negative effect onto endogenous Igfbp-1 gene expression in SV40-transformed hepatocytes.

The LXXLL motif of FoxO1 is indispensable for binding to Sirt1 in vivo. From previous studies, the LXXLL motif of FoxO1 appears to be quite important for its transcriptional activity. Therefore, we sought to identify the mechanism by which the LXXLL motif 


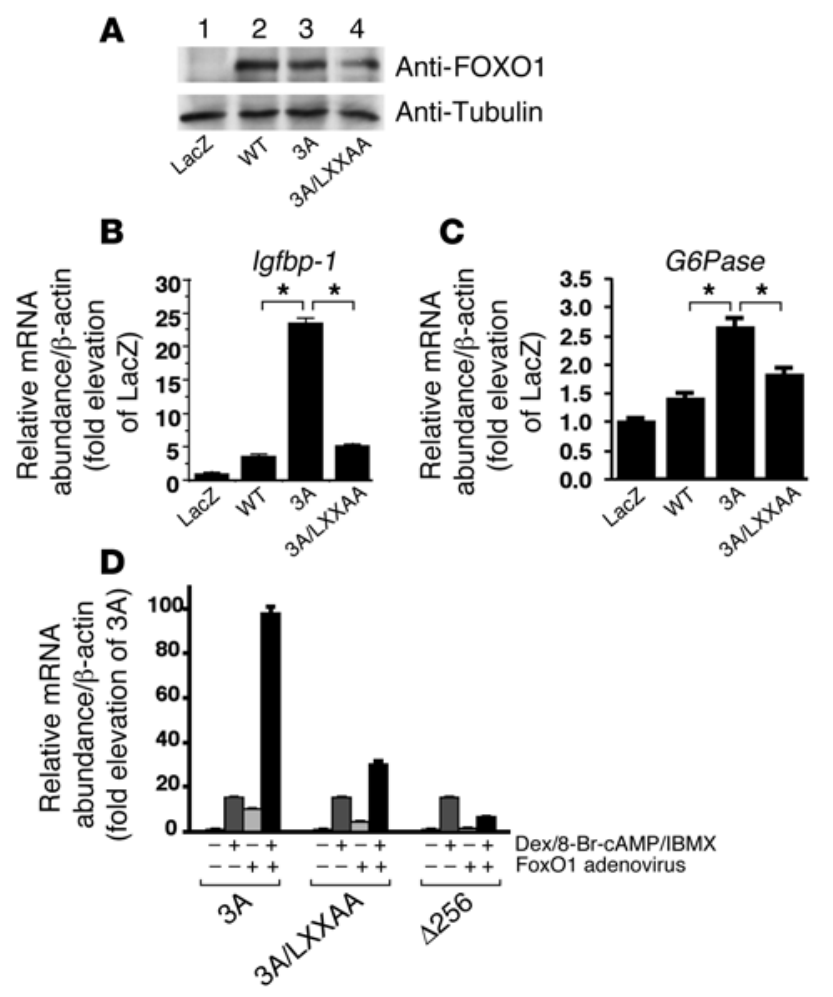

of FoxO1 is involved in its transcriptional regulation. The nicotinamide adenine dinucleotide-dependent (NAD-dependent) deacetylase Sirt1 is abundantly expressed in SV40-transformed hepatocytes (Figure 5A). After transduction of SV40-transformed hepatocytes with adenovirus vector encoding $\mathrm{LacZ}$, 3A FoxO1, or 3A/LXXAA FoxO1, we immunoprecipitated 3A FoxO1 or 3A/LXXAA FoxO1 using anti-Flag antibody and blotted with anti-silent information regulator 2 (anti-Sir2; mammalian Sirt1) antibody. We showed that $3 \mathrm{~A}$ FoxO1 bound to endogenous Sirt1. However, 3A/LXXAA FoxO1 failed to do so (Figure 5A). Furthermore, immunoprecipitation with anti-Sir 2 antibody revealed that endogenous Sirt1 interacted with transduced Flag 3A FoxO1 but not with Flag 3A/LXXAA FoxO1 (Figure 5A). These data suggest that the LXXLL motif of FoxO1 is important for binding to Sirt1.

In order to investigate the effects of disruption of the LXXLL motif of FoxO1 on its acetylation, we performed Western blotting with anti-acetylated lysine antibody using the same sample. Mutagenesis of the LXXLL motif in 3A FoxO1 showed increased acetylation compared with that of $3 \mathrm{~A}$ FoxO1 (Figure 5, B and C). Furthermore, in order to investigate effects of disruption of the LXXLL

\section{Figure 4}

Effects of $3 A / L X X A A$ FoxO1 on endogenous /gfbp-1 gene expression in SV40-transformed hepatocytes. (A) Expression of Flag 3A/LXXAA FoxO1 and HA-ADA FoxO1 at indicated MOI in SV40-transformed hepatocytes. (B) The effects of 3A/LXXAA FoxO1 on HA-ADA-induced Igfbp-1 gene expression in the presence of dexamethasone/8- $\mathrm{Br}$ cAMP/IBMX in SV40-transformed hepatocytes. Data from 3 independent experiments are expressed as mean \pm SEM of the folds of endogenous Igfbp-1 gene expression in the absence of HA-ADA FoxO1. *Statistically significant difference between 3A/LXXAA FoxO1 at MOI 0 and at MOI 10 or 20. $P<0.001$ by 1 -way ANOVA.

\section{Figure 3}

Effects of 3A FoxO1 and 3A/LXXAA FoxO1 mutants on endogenous Igfbp-1 or G6Pase gene expression in SV40-transformed hepatocytes. (A) Expression of HA-WT, Flag 3A FoxO1, or 3A/LXXAA FoxO1 in SV40-transformed hepatocytes was detected. Lane 1, hepatocytes transduced with adenovirus encoding LacZ; lane 2, with WT; lane 3, with $3 \mathrm{~A}$ mutant; lane 4 , with $3 \mathrm{~A} / \mathrm{LXXAA}$ mutant at $10 \mathrm{MOI}$. Endogenous Igfbp-1 (B) or G6Pase (C) gene expression in SV40-transformed hepatocytes transduced with adenovirus encoding LacZ, WT FoxO1, 3A FoxO1, or 3A/LXXAA FoxO1. Hepatocytes transduced with each adenovirus were incubated in serum-free medium overnight before addition of dexamethasone/8-Br-cAMP/IBMX and incubation for 8 hours. Isolation of total RNA and real-time PCR were performed as described in Methods. Data (mean \pm SEM) are from 3 independent experiments and are normalized by the amount of $\beta$-actin mRNA and expressed relative to the corresponding LacZ value. *Statistically significant difference between $3 A$ - and WT- or 3A/LXXAA-transduced cells at each MOI ( $P<0.001$ by 1 -way ANOVA). (D) Effects of 3A FoxO1, 3A/LXXAA FoxO1, and $\Delta 256$ FoxO1 mutants on endogenous Igfbp-1 gene expression in SV40-transformed hepatocytes. Hepatocytes transduced with each adenovirus at $10 \mathrm{MOI}$ were incubated in serum-free medium overnight before addition of dexamethasone/8-Br-cAMP/IBMX for 8 hours. Isolation of total RNA and real-time PCR were performed as described in Methods. Data are from 3 independent experiments and are expressed as mean \pm SEM of fold of relative gene expression for $\beta$-actin in LacZ-transduced hepatocytes in the absence of dexamethasone/8-Br-cAMP/IBMX. motif on acetylation of FoxO1 in the presence of nicotinamide, an inhibitor of Sirt1, and/or trichostatin A (TSA), an inhibitor of class I and class II histone deacetylases (HDACs), we transfected HEK293 cells with pCMV5/cMyc/3A FoxO1 or 3A/LXXAA FoxO1, immunoprecipitated cell lysates with anti-cMyc antibody, and blotted with anti-acetylated lysine polyclonal antibody. Although acetylation of 3A/LXXAA FoxO1 was detected in the presence of nicotinamide and TSA (Figure 5D), 3A FoxO1 was not acetylated under the same conditions (Figure 5D). These data suggest that disruption of the LXXLL motif enhances acetylation of FoxO1.

Sirt1 inhibitor inhibits Igfbp-1 and G6Pase promoter activity in an LXXLL motif-dependent manner. Nicotinamide is a Sirt1-specific deacetylase inhibitor. The effects of Sirt 1 on transcriptional activity of FoxO family members are controversial. These effects seem to be

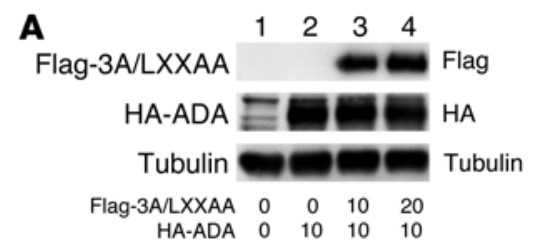

B

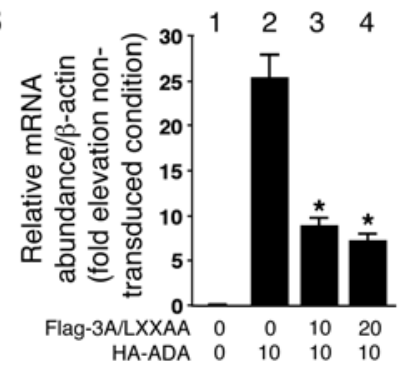


A

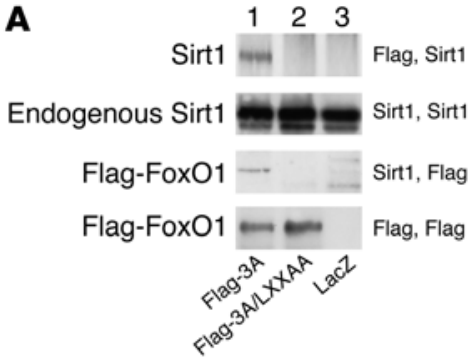

\section{B}

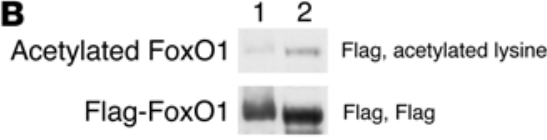

D

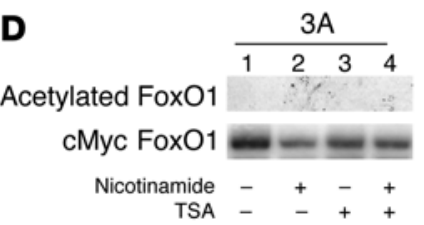

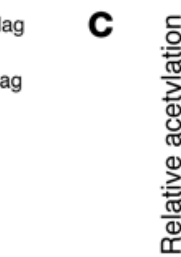

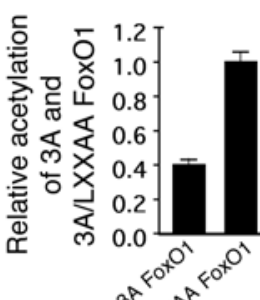

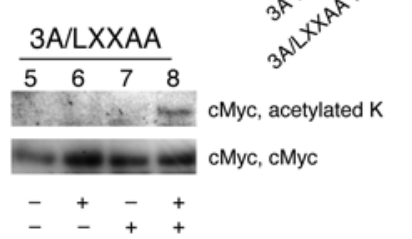

\section{Figure 5}

Sirt1 interacts with 3A FoxO1 but not with 3A/LXXAA FoxO1, and disruption of the LXXLL motif enhances acetylation of FoxO1. (A) SV40-transformed hepatocytes were transduced with adenovirus encoding Flag 3A FoxO1 (lane 1) or Flag 3A/LXXAA FoxO1 (lane 2). After 24 hours serum starvation, cells were cultured in AMEM supplemented with $0.1 \%$ bovine serum albumin, $0.5 \mathrm{mM}$ 8-Br-cAMP, $0.5 \mathrm{mM} I \mathrm{IBMX}$, and $1 \mu \mathrm{M}$ dexamethasone for 8 hours. Lysates were immunoprecipitated using anti-Flag monoclonal antibody (M2) and Western blotted with anti-Sir2 polyclonal (first panel) or anti-Flag monoclonal antibody (M2) (fourth panel) or immunoprecipitated using anti-Sir2 polyclonal antibody and Western blotted with anti-Sir2 antibody (second panel) or anti-Flag monoclonal antibody (third panel). (B) SV40transformed hepatocytes transduced with adenovirus encoding Flag 3A FoxO1 (lane 1) or Flag 3A/LXXAA FoxO1 (lane 2) were incubated with $0.5 \mathrm{mM}$ 8-Br-cAMP, $0.5 \mathrm{mM}$ IBMX, and $1 \mu \mathrm{M}$ dexamethasone for 8 hours after 24 hours of serum starvation. Lysates were immunoprecipitated using anti-Flag monoclonal antibody (M2) and Western blotted with anti-acetylated lysine polyclonal (top panel) or anti-Flag monoclonal antibody (M2) (bottom panel). (C) Quantification of the data in B. Mean \pm SEM of the folds of acetylation of $3 \mathrm{~A} /$ LXXAA was calculated from 3 independent experiments using NIH Image 1.62 (http://rsb. info.nih.gov/nih-image/). Subsequent blotting with anti-Flag antibody using the same filter normalized acetylation of each FoxO1. (D) HEK293 cells transfected with pCMV5/cMyc/3A FoxO1 (lanes 1-4) or 3A/LXXAA FoxO1 (lanes 5-8) were incubated for 8 hours in the absence or presence of nicotinamide $(50 \mathrm{mM})$ or trichostatin $\mathrm{A}$ (TSA) $(2 \mu \mathrm{M})$. cMyc-tagged FoxO1 was immunoprecipitated, and the acetylation of FoxO1 was assessed by Western blot with the antibody to acetylated lysine (upper panel). Total levels of FoxO1 were assessed with the antibody to cMyc (lower panel).

dependent on FoxO's target genes (27-30). In order to investigate whether the LXXLL motif of FoxO1 mediates Sirt1-dependent promoter activity of FoxO1 target genes, promoter assay of Igfbp-1 or G6Pase was performed. Nicotinamide decreased Igfbp-1 promoter activity by $48 \%$ compared with promoter activity induced by both dexamethasone/8-Br-cAMP/IBMX and 3A FoxO1 (Figure 6A). In contrast, nicotinamide did not change Igfbp-1 promoter activity induced by both dexamethasone/8-Br-cAMP/IBMX and 3A/ LXXAA FoxO1 (Figure 6A). Furthermore, nicotinamide decreased G6Pase promoter activity by $70 \%$ compared with promoter activity induced by both dexamethasone/8-Br-cAMP/IBMX and 3A FoxO1 (Figure 6B). However, nicotinamide did not change G6Pase promoter activity induced by both dexamethasone/8-Br-cAMP/IBMX and 3A/LXXAA FoxO1 (Figure 6B). Interestingly, Igfbp-1 and G6Pase promoter activity induced by both dexamethasone/8-Br-cAMP/ IBMX and 3A/LXXAA FoxO1 was the same as the nicotinamidetreated promoter activity level of 3A FoxO1 (Figure 6, A and B). In order to confirm the effect of the Sirt1 inhibitor on transcrip-

tional regulation of FoxO1, we used another Sirt1 inhibitor, BML-210 (27), and performed the same promoter assays. It has been reported that BML-210 was a less potent inhibitor of Sirt1 than nicotinamide (27). BML-210 significantly decreased Igfbp-1 promoter activity (by $25 \%$ ) compared with promoter activity induced by both dexamethasone/8-Br-cAMP/IBMX and $3 \mathrm{~A}$ FoxO1 (Figure 7A). In contrast, BML-210 did not change I $g f b p-1$ promoter activity induced by both dexamethasone/8-Br-cAMP/IBMX and 3A/LXXAA FoxO1 (Figure 7A). Furthermore, BML-210 significantly decreased G6Pase promoter activity (by 17\%) compared with promoter activity induced by both dexamethasone/8-BrcAMP/IBMX and 3A FoxO1 (Figure 7B). However, BML-210 did not change G6Pase promoter activity induced by both dexamethasone/8-BrcAMP/IBMX and 3A/LXXAA FoxO1 (Figure 7B). These data demonstrate that nicotinamide can inhibit 3A FoxO1-induced promoter activity of Igfbp-1 and G6Pase to the same level as that induced by $3 \mathrm{~A} / \mathrm{LXXAA}$ FoxO1 and suggest that the LXXLL motif mediates the Sirt1-dependent transcriptional activity of FoxO1.

Resveratrol activates Igfbp-1 and G6Pase promoter activity in an LXXLL motif-dependent manner. Resveratrol was found to be a potent activator of Sirt1 (31). Recently, it has been reported that resveratrol increases glucose production by increasing FoxO1-dependent transcription of gluconeogenic genes in H4IIE rat hepatoma cells (32). In order to determine whether the LXXLL motif in FoxO1 is involved in Sirt1-dependent transcriptional regulation, we also assessed effects of resveratrol on Igfbp-1 or G6Pase promoter activity induced by both dexamethasone/ 8-Br-cAMP/IBMX and 3A FoxO1 or 3A/LXXAA FoxO1. Resveratrol increased Igfbp-1 or G6Pase promoter activity induced by both dexamethasone/8-Br-cAMP/IBMX and 3A FoxO1 by $30 \%$ and $37 \%$, respectively $(P<0.005$ or $P<0.001$ by 1-way ANOVA, respectively) (Figure 8, A and B). In contrast, resveratrol had no effects on both Igfbp-1 and G6Pase promoter activity levels induced by both dexamethasone/8-Br-cAMP/IBMX and 3A/ LXXAA FoxO1 (Figure 8, A and B). These data suggest that resveratrol, a Sirt 1 activator, activates promoter activity of FoxO1 target genes in an LXXLL motif-dependent manner.

Effects of acute hepatic overexpression of $3 A / L X X A A$ FoxO1 on glucose metabolism in Leprdb/db mice. From the previous data, it appears that $3 \mathrm{~A} / \mathrm{LXXAA}$ FoxO1 acts as a dominant-negative type FoxO1. In order to confirm the dominant-negative effect of $3 \mathrm{~A} / \mathrm{LXXAA}$ FoxO1 in vivo, we administered adenovirus $\left(4 \times 10^{8} \mathrm{pfu} / \mathrm{g}\right.$ of body weight) encoding LacZ, 3A FoxO1, or 3A/LXXAA FoxO1 into leptin receptor-deficient Leprdb/db mice (Figure 9, A and B). These mice show hyperphagia, obesity, and insulin resistance in their basal state, and it has already been reported that about $50 \%$ of total intracellular FoxO1 proteins were localized in nucleus in livers from Leprdb/db mice (33). Therefore, it is appropriate to use $L e p^{d b / d b}$ mice for investigation of a dominant-negative effect of 


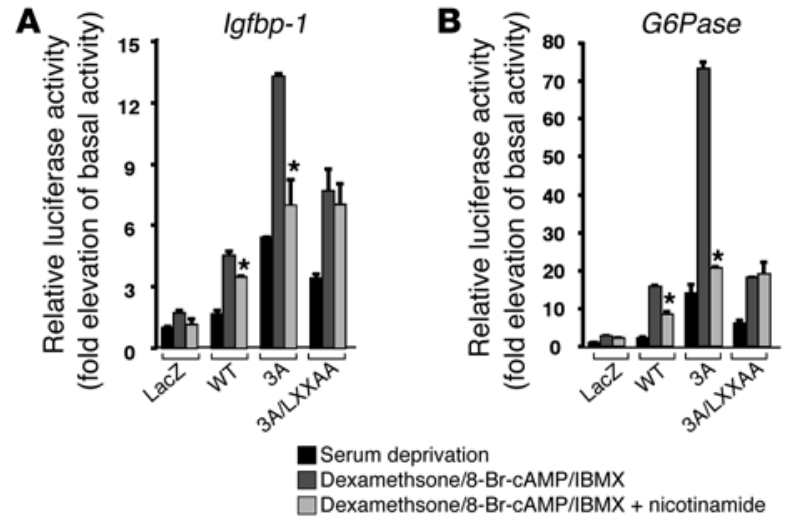

3A/LXXAA FoxO1. Immunohistochemistry using anti-FoxO1 serum demonstrated that both exogenous FoxO1 mutants were localized in nucleus of liver (Figure 9B). Five days after injection, we performed intraperitoneal glucose tolerance tests. Fasting blood glucose levels of mice injected with $3 \mathrm{~A}$ FoxO1 increased significantly compared with those of mice injected with adenovirus encoding LacZ ( $469.6 \pm 30.7$ versus $311 \pm 27.5$; $P<0.001$ by 1 -way ANOVA). In contrast, fasting blood glucose of mice injected with 3A/LXXAA FoxO1 decreased significantly compared with mice injected with $\mathrm{LacZ}$ or $3 \mathrm{~A}$ FoxO1 $(176 \pm 30.7$ versus $311 \pm 27.5$ or $469.9 \pm 30.7 ; P<0.001$ by 1 -way ANOVA) (Figure 9C). Furthermore, intraperitoneal glucose tolerance testing showed that mice injected with 3A/LXXAA FoxO1 were significantly more glucose tolerant than mice injected with $\mathrm{LacZ}$ or 3A FoxO1 (Figure 9D). These data suggest that overexpression of 3A/LXXAA FoxO1 in liver can alleviate insulin resistance of $L e p r^{d b / d b}$ mice.

Overexpression of 3A/LXXAA FoxO1 in liver from $L e p r^{d b / d b}$ mice inhibits glucose production through inhibition of G6Pase gene expression. Real-time PCR shows that G6Pase and Igfbp-1 gene expression levels in liver from fasted mice injected with $3 \mathrm{~A} /$ LXXAA FoxO1 are significantly decreased compared with those in mice injected with $3 \mathrm{~A}$ FoxO1. In contrast, Pepck gene expression level was not different statistically among LacZ, 3A FoxO1, and 3A/LXXAA FoxO1-treated mice (Figure 10A). In order to confirm whether both 3A FoxO1 and 3A/LXXAA FoxO1 can bind to promoter regions of FoxO1 target genes, which include $I g f b p-1$, G6Pase, and Pepck, chromatin immunoprecipitation (ChIP) assay was performed using liver from Lepr ${ }^{d b / d b}$ mice injected with

\section{Figure 7}

BML-210 inhibits FoxO1-dependent induction of a reporter construct under the control of an Igfbp-1 or G6Pase promoter. After hepatocytes were transfected with Igfbp-1/(p925GL3) (A) or G6Pase/luciferase reporter gene (PicaGene/human G6Pase promoter-luciferase) (B), cells were infected with indicated adenovirus. phRL-SV40 was used as an internal control of transfection efficiency. After overnight serum deprivation (black bars) and induction with dexamethasone/8- $\mathrm{Br}$ cAMP/IBMX as described in Methods in the presence (light gray bars) or absence (dark gray bars) of BML-210 $(1.5 \mathrm{mM})$, cells were harvested and luciferase activity was measured. * ${ }^{*}$ tatistically significant difference between luciferase activity in the absence and luciferase activity in the presence of BML-210 at cells transfected with each vector, $P<0.01$ by 1 -way ANOVA. Data represent mean \pm SEM from 3 independent experiments.

\section{Figure 6}

Nicotinamide inhibits FoxO1-dependent induction of a reporter construct under the control of an Igfbp-1 or G6Pase promoter. After hepatocytes were transfected with Igfbp-1/(p925GL3) (A) or G6Pase/luciferase reporter gene (PicaGene/human G6Pase promoter-luciferase) (B), cells were infected with indicated adenovirus. phRL-SV40 was used as an internal control of transfection efficiency. After overnight serum deprivation (black bars) and induction with dexamethasone/8-Br-cAMP/ IBMX as described in Methods in the presence (light gray bars) or absence (dark gray bars) of nicotinamide (50 mM), cells were harvested and luciferase activity was measured. *Statistically significant difference between luciferase activity in the absence and in the presence of nicotinamide in cells transfected with each vector. $P<0.005$ by 1 -way ANOVA. Data represent mean \pm SEM from 3 independent experiments.

adenovirus. Both 3A FoxO1 and 3A/LXXAA FoxO1 bound to promoter regions of Igfbp-1 and G6Pase (Figure 10B). Interestingly, although FoxO1 didn't affect Pepck gene expression in liver in the present study, both 3A FoxO1 and 3A/LXXAA FoxO1 can bind to Pepck promoter region (Figure 10B). Furthermore, hepatic glycogen content of mice injected with 3A/LXXAA FoxO1 increased significantly compared with mice injected with the LacZ or $3 \mathrm{~A}$ FoxO1 $(0.0162 \pm 0.0014$ versus $0.0071 \pm 0.0008$ or $0.0065 \pm 0.0009 \mathrm{mg}$ glycogen/mg liver, respectively, $P<0.01$ by 1 -way ANOVA) (Figure $10 \mathrm{C})$. These data suggest that $3 \mathrm{~A} / \mathrm{LXXAA}$ FoxO1 can bind to the promoter region of FoxO1 target genes in liver and that acute overexpression of 3A/LXXAA FoxO1 inhibits G6Pase gene expression and suppresses glucose production in liver from $L e p r^{d b / d b}$ mice.

Disruption of the LXXLL motifenhances acetylation of FoxO1 in liver. As described above, we demonstrated that disruption of the LXXLL motif inhibits binding of FoxO 1 to Sirt 1 and enhances acetylation of FoxO1 in SV40-transformed hepatocytes or HEK293 cells. In order to confirm effects of disruption of the LXXLL motif in FoxO1 on in vivo acetylation in liver, we performed immunoprecipitation of liver lysates injected with adenovirus encoding 3A FoxO1 or 3A/LXXAA FoxO1 using anti-Flag antibody and Western blotting using anti-acetylated lysine antibody. Although both $3 \mathrm{~A}$ FoxO1 and 3A/LXXAA FoxO1 can be coimmunoprecipitated with endogenous $\mathrm{Cbp}$ and Pgc-1 $\alpha, 3 \mathrm{~A} / \mathrm{LXXAA}$ FoxO1 binds to endogenous Sirt 1 weakly compared with $3 \mathrm{~A}$ FoxO1 (Figure 11). Consistent with data described above, acetylation of 3A/LXXAA FoxO1 is enhanced compared with that of $3 \mathrm{~A}$ FoxO1 (Figure 11). These data suggest that disruption of the LXXLL motif in FoxO1 doesn't affect bind-
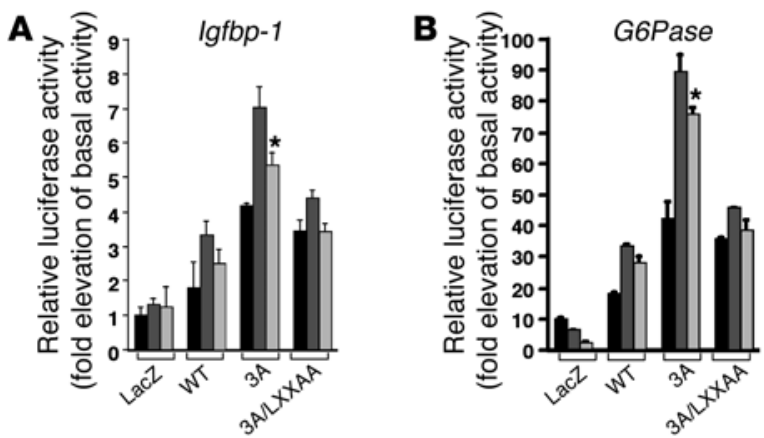

Serum deprivation Dexamethsone/8-Br-cAMP/IBMX $\square$ Dexamethsone/8-Br-cAMP/BMX + BML-210 


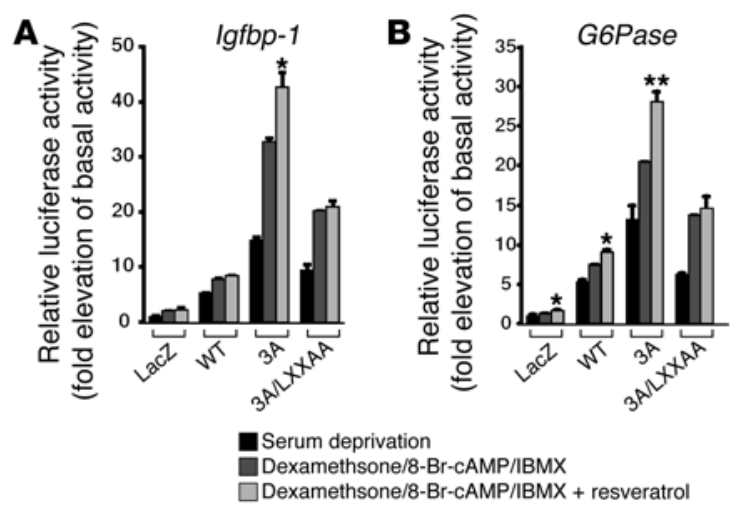

ing to $\mathrm{Cbp}$ but inhibits interaction with Sirt 1 and finally enhances acetylation of FoxO1 in vivo.

\section{Discussion}

The present data reveal that the LXXLL motif of FoxO1 has an important role for its transcriptional activity. We show here that (a) the LXXLL motif of FoxO proteins is conserved among different species; (b) disruption of the LXXLL motif of FoxO1 abolishes transcriptional activity on the Igfbp-1 or G6Pase promoter; (c) disruption of the LXXLL motif inhibits dexamethasone/8-Br-cAMP/ IBMX-induced Igfbp-1 and G6Pase gene expression in SV40-transformed hepatocytes; (d) disruption of the LXXLL motif abolishes the interaction between FoxO1 and Sirt 1 and enhances acetylation of a constitutively active mutant $3 \mathrm{~A}$ FoxO1; (e) disruption of the LXXLL motif makes FoxO1 nicotinamide or resveratrol insensitive; and (f) overexpression of 3A/LXXAA FoxO1 in liver of Lepr db/db mice ameliorates insulin resistance through inhibition of G6Pase gene expression and suppression of glucose production. These results might be especially relevant to involvement of the LXXLL motif of FoxO1 in control of target gene expression of FoxO1.

The substitution of alanine for 3 Akt phosphorylation sites (T24, S253, and S316) yields a constitutively nuclear mutant FoxO1 that is in the nucleus even in the presence of serum (32). Disruption of the LXXLL motif by substitution of leucine residues at amino acids 462 and 463 with alanine residues does not change subcellular localization of $3 \mathrm{~A}$ FoxO1 mutant in SV40-transformed hepatocytes. Furthermore, even in liver transduced with adenovirus encoding 3A/LXXAA FoxO1, immunohistochemistry using anti-FoxO1 antibody demonstrated that 3A/LXXAA FoxO1 as well as $3 \mathrm{~A}$ FoxO1 was localized in the nucleus. ChIP assay also showed that 3A/LXXAA FoxO1 could bind to promoter regions of FoxO1 target genes. However, 3A/LXXAA FoxO1 cannot activate transcription of its target genes, such as Igfbp-1 and G6Pase. These data indicate that $3 \mathrm{~A} / \mathrm{LXXAA}$ FoxO1 can compete with endogenous FoxO family members in liver and has a dominant-negative effect on transcription of FoxO1 target genes because it has been reported that endogenous FoxO1 in liver from Leprdb/db mice was localized in nucleus (33). Indeed, we demonstrated that overexpression of 3A/LXXAA FoxO1 inhibited endogenous Igfbp-1 gene expression induced by constitutively nuclear HA-ADA FoxO1 in a dose-dependent manner in SV40-transformed hepatocytes.

Sir2 is an NAD-dependent $\operatorname{HDAC}(34,35)$. Mutant strains of C. elegans with extra copies of Sir2.1 have increased longevity, and Daf16 (C. elegans FoxO) is epistatic to Sir2.1 gain of function (36). Sirtuins, the 7 mammalian Sir orthologs, can deacetylate transcrip-

\section{Figure 8}

Resveratrol activates induction of a reporter construct under the control of an Igfbp-1 or G6Pase promoter. After hepatocytes were transfected with Igfbp-1/(p925GL3) (A) or G6Pase/luciferase reporter gene (PicaGene/human G6Pase promoter-luciferase) (B), cells were infected with indicated adenovirus. phRL-SV40 was used as an internal control of transfection efficiency. After overnight serum deprivation (black bars) and induction with dexamethasone/8-Br-cAMP/IBMX as described in Methods in the presence (light gray bars) or absence (dark gray bars) of resveratrol $(10 \mu \mathrm{M})$, cells were harvested and luciferase activity was measured. *Statistically significant difference between luciferase activity in the absence and luciferase activity in the presence of resveratrol, ${ }^{*} P<0.005$ and ${ }^{* *} P<0.001$ by 1 -way ANOVA. Data represent mean \pm SEM from 3 independent experiments.

tion factors, such as p53 (37-40), MyoD (41), and FoxOs (27-30). FoxOs are acetylated in response to cellular stress by the acetyltransferase activity of the nuclear hormone receptor coactivators $\mathrm{Cbp}$ and $\mathrm{p} 300$. Sirt 1 can deacetylate $\mathrm{Cbp}$-acetylated FoxO proteins (27-30,37-42). Recently, it has been reported that Sirt1 is involved in induction of gluconeogenic genes in liver through deacetylation of Pgc-1 $\alpha$ (43) or of FoxO1 (32). Therefore, Sirt1 has an important role in glucose metabolism. The present study demonstrates that disruption of the LXXLL motif of FoxO1 inhibits interactions with Sirt 1 and FoxO1 deacetylation in cell lines as well as liver. We also showed that 3A/LXXAA FoxO1 is still acetylated in the presence of both nicotinamide and trichostatin in HEK293 cells incubated with $\mathrm{H}_{2} \mathrm{O}_{2}$. However, $3 \mathrm{~A}$ FoxO1 was deacetylated even in the presence of both nicotinamide and trichostatin. These data suggest the possibility that both Sirt 1 and class I and II HDACs contribute to FoxO1 deacetylation in vivo and are consistent with previous reports (27). In the present study, nicotinamide was shown to significantly inhibit both Igfbp-1 and G6Pase promoter activity induced by dexamethasone/8-Br-cAMP/IBMX and 3A FoxO1. However, nicotinamide did not change promoter activity of both genes induced by dexamethasone/8-Br-cAMP/IBMX and 3A/LXXAA FoxO1. Interestingly, promoter activity level inhibited by nicotinamide is the same as induced by dexamethasone/8-Br-cAMP/IBMX and 3A/LXXAA FoxO1. In contrast, another Sirt 1 inhibitor, BML210 , which has been reported to be a less potent Sirt 1 inhibitor (27), could weakly inhibit Igfbp-1 and G6Pase promoter activity induced by dexamethasone/8-Br-cAMP/IBMX and 3A FoxO1 compared with nicotinamide. These data suggest that activity of Sirt 1 may affect transcriptional activity of FoxO1. Resveratrol is among a number of small molecules that were initially shown to activate the catalytic activity of SIRT1 and yeast Sir2 protein (31). Recent reports suggest that the activation of Sir2 by resveratrol may result from the fluorescently tagged substrate used in the in vitro assay for Sir2 deacetylase activity $(44,45)$. Moreover, it has been reported that resveratrol extends life span by inhibiting Sir2-mediated repression of ER stress genes (46). Still there is controversy about the mechanism by which resveratrol activates Sirt 1 activity. However, in the present study, resveratrol increased promoter activity of Igfbp-1 or G6Pase induced by dexamethasone/8-Br-cAMP/IBMX and $3 \mathrm{~A}$ FoxO 1 by $30 \%$ or $40 \%$, respectively. In contrast, resveratrol had no effects on Igfbp-1 and G6Pase promoter activity induced by dexamethasone/8-Br-cAMP/IBMX and 3A/LXXAA FoxO1. These data suggest that disruption of the LXXLL motif makes FoxO1 nicotinamide or resveratrol insensitive and that $3 \mathrm{~A} / \mathrm{LXXAA}$ FoxO1 has a dominant-negative effect on coordination of FoxO1-and Sirt1- 

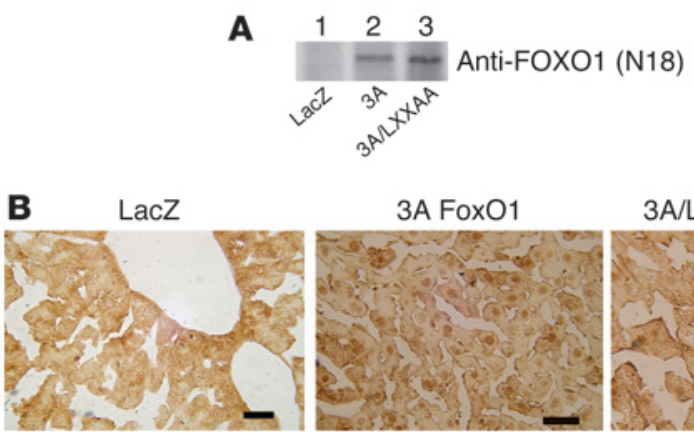

3A/LXXAA FoxO1
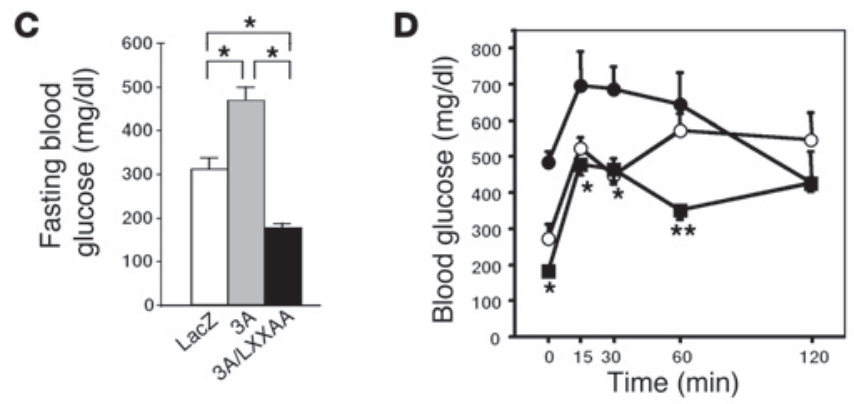

Figure 9

Effects of overexpression of 3A/LXXAA FoxO1 on fasted blood glucose and glucose tolerance in Leprdb/db mice. (A) Expression of 3A FoxO1 or 3A/LXXAA FoxO1 mutant in liver. Liver was removed from mice at day 5 after injection with adenovirus encoding LacZ (lane 1), 3A FoxO1 (lane 2), or 3A/LXXAA FoxO1 (lane 3) intravenously, and $15 \mu \mathrm{g}$ of each liver lysate was subjected to immunoblot analysis with an anti-FOXO1 polyclonal antibody (N18). (B) Immunohistochemistry of liver from Lepr ${ }^{d b / d b}$ mice injected with adenovirus encoding LacZ (left panel), 3A FoxO1 (middle panel), or 3A/LXXAA FoxO1 (right panel). Each panel shows representative sections. Magnification, $\times 40$. Scale bar: $10 \mu \mathrm{m}$. (C) Effect of overexpression of $3 \mathrm{~A}$ FoxO1 or 3A/LXXAA FoxO1 in liver from Leprdb/db mice on fasted blood glucose. Blood glucose levels were measured as described in Methods after overnight fasting at day 5 after injection with each adenovirus encoding LacZ (white bar), 3A FoxO1 (gray bar) or 3A/LXXAA FoxO1 (black bar). Data represent mean \pm SEM of 6 mice for each group. *Statistically significant difference between mice injected with each adenovirus encoding LacZ, 3A FoxO1, or 3A/LXXAA FoxO1. $P<0.001$ by 1 -way ANOVA. (D) Intraperitoneal glucose tolerance test was performed in mice injected with the adenoviruses indicated. The groups of mice indicated were injected with a $1.2 \mathrm{~g} / \mathrm{kg}$ of body weight of glucose intraperitoneally as follows: LacZ (open circles, $n=6$ ), 3A FoxO1 (filled circles, $n=6$ ), and 3A/LXXAA FoxO1 (filled squares, $n=6$ ). *Statistically significant difference between $3 \mathrm{~A}$ and 3A/LXXAA, $P<0.01$ by 1 -way ANOVA; ** statistically significant difference between $3 A / L X X A A$ and LacZ or $3 A, P<0.01$ by 1 -way ANOVA.

specific transcriptional activities; they also support the previous report that FoxO1 deacetylation is indispensable for full transcriptional activation of Igfbp-1 (47) and G6Pase.

Pgc- $1 \alpha$ is deacetylated by Sirt 1 (43) and also binds to FoxO1 (48). There is a possibility that the LXXLL motif of FoxO1 may affect binding to Pgc-1 $\alpha$. However, in the present study, we could not detect any differences of binding to Pgc- $1 \alpha$ between $3 \mathrm{~A} \mathrm{FoxO} 1$ and 3A/LXXAA FoxO1 using liver lysates. Indeed, it has been reported that the binding site of Pgc- $1 \alpha$ is located in the $\mathrm{N}$ terminus of FoxO1, which includes the forkhead DNA-binding domain (48). Furthermore, although it has been suggested that the LXXLL motif of the FoxO family may be important for binding to $\mathrm{Cbp} /$ p300 (49), we demonstrated that disruption of the LXXLL motif did not affect binding to Cbp in this study. Recent studies suggested phosphorylation and interaction of DAF-16, which is a homolog of FoxO1 in C. elegans, with 14-3-3 might be important for the formation of the Sir2.1/DAF-16 complex (50). Therefore, it is possible to speculate that an unknown molecule that specifically binds to the LXXLL motif of FoxO1 may affect binding between FoxO1 and Sirt1.

The present study shows that the LXXLL motif of FoxO1 is involved in gene expression of FoxO1 target genes, such as Igfbp-1 and G6Pase, through interaction with Sirt 1 and suggest that the LXXLL motif should be a target site for regulation of gene expression of FoxO1 target genes and for treatment of type 2 diabetes.

\section{Methods}

Antibodies. We purchased anti-Flag (M2) and anti-tubulin monoclonal antibodies from Sigma-Aldrich, anti-Sir2 from Upstate USA Inc., acetylated-lysine polyclonal antibodies from Cell Signaling Technology, and anti-FOXO1 polyclonal antibodies (H128 and N18) and anti-PGC-1 $\alpha$ polyclonal antibodies from Santa Cruz Biotechnology Inc.

Construction of expression vectors and adenoviral vectors. For mutagenesis of FoxO1, we performed overlap extension PCR using pCMV5/ cMyc/WT FoxO1 as a template as described previously (25). For construction of pCMV5/cMyc/T24A/S253A/S316A (3A FoxO1), the following mutagenic primers were used: primer 5, 5'-AGAGCTGCGGCCATGGACAAC-3', corresponding to nucleotides 844-864; and primer 6, 5'-GTTGTCCATGGCCGCAGCTCT-3', corresponding to nucleotides 864-844. For construction of 3A/LXXAA FoxO1, we used the following: primer 1, 5'-TTGAAAGAGGCGGCGACTTCTGAC-3', corresponding to nucleotides 1381-1404; and primer 2, 5'-GTCAGAAGTCGCCGCCTCTTTCAA-3', corresponding to nucleotides $1404-1381$ as mutagenic primers. We used pCMV5/cMyc/3A FoxO1 as a PCR template.

For construction of adenoviral vectors, we used a Flag epitope tag. The XbaI-treated 3A FoxO1 or 3A/LXXAA FoxO1 fragment was subcloned into XbaI-treated pFLAG CMV-2 Expression Vector (SigmaAldrich). DNA encoding Flag 3A FoxO1 or 3A/LXXAA FoxO1 was subcloned into Nhe I-treated pShuttle2 vector (Clontech). After confirming protein expression in SV40-transformed hepatocytes by transient transfection with pShuttle2/Flag 3A FoxO1 or Flag 3A/LXXAA FoxO1 vector, I-CeuI- and PI-SceI-treated fragment was subcloned into Adeno-X viral DNA (Clontech). Adenovirus vector containing these cDNAs was generated by transfecting HEK293 cells with the corresponding pAdeno-XTM plasmid.

Cell culture, transfection, and viral transduction. SV40-transformed hepatocytes used in these studies have been described in previous work (25). For induction of endogenous Igfbp-1, G6Pase, and Pepck gene expression, we infected hepatocytes with adenoviral vector at MOIs between 10 and 20. At 24 hours after transduction, cells were serum starved overnight and then cultured with $\alpha$-modified Eagle's medium (AMEM; Invitrogen) supplemented with $0.1 \%$ bovine serum albumin, $0.5 \mathrm{mM} 8-\mathrm{Br}$ cAMP (Sigma-Aldrich), $0.5 \mathrm{mM}$ IBMX (Sigma-Aldrich), and $1 \mathrm{mM}$ dexamethasone (Sigma-Aldrich) for 8 hours.

Luciferase assay. Transfection was carried out on cells at a 70\%-80\% confluence using $1.5 \mu \mathrm{g}$ of Igfbp-1/luciferase reporter gene (p925GL3) or PicaGene/human G6Pase promoter-luciferase (51). Synthetic Renilla luciferase reporter vector (phRL-SV40; Promega) (10 ng) was used as an internal control of transfection efficiency. After transient transfection, cells were infected with adenovirus indicated at MOIs between 10 and 20. At 24 hours after transduction, overnight serum starvation and induction with $0.5 \mathrm{mM}$ 

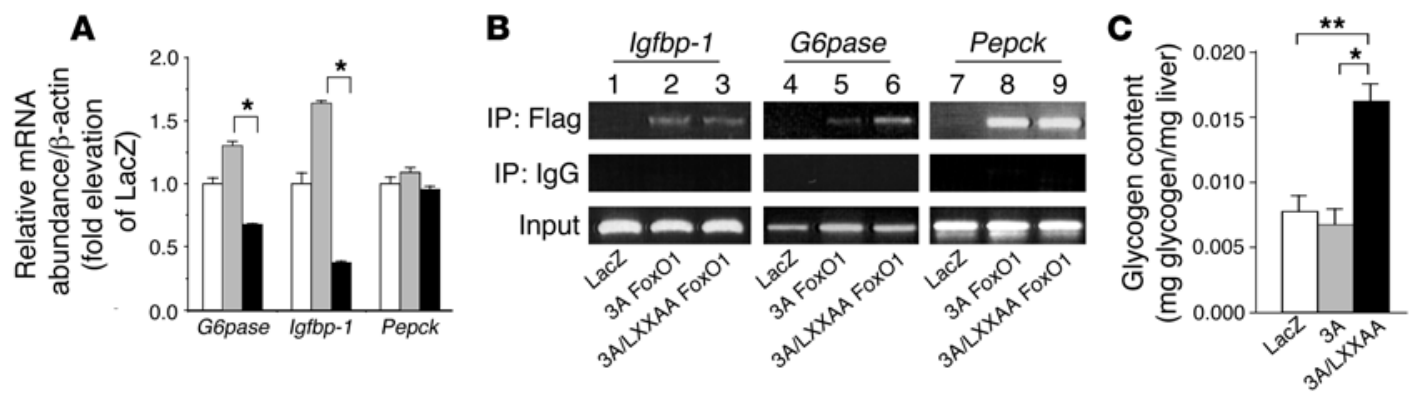

\section{Figure 10}

Effects of overexpression of 3A/LXXAA FoxO1 in liver on gene expression and hepatic glycogen content. (A) Real-time PCR. Following overnight fasting, mRNA from the liver of Leprdb/db mice injected with adenovirus encoding LacZ (white bars), 3A FoxO1 (gray bars), or 3A/LXXAA FoxO1 (black bars) was isolated at day 5 after injection. Real-time PCR was performed with primers encoding the genes indicated. Data represent mean \pm SEM of 3 independent experiments ( $n=4$ for each group). *Statistically significant difference between 3A FoxO1 and 3A/LXXAA FoxO1, $P<0.02$ by 1 -way ANOVA; ${ }^{* *}$ statistically significant difference between LacZ and 3 A FoxO1, $P<0.05$ by 1 -way ANOVA. (B) ChIP assay of Igfbp-1 (lanes 1-3), G6Pase (lanes 4-6), and Pepck (lanes 7-9) promoter. ChIP assay was performed using liver sections from Leprdb/db mice injected with adenovirus encoding LacZ, 3A FoxO1, or 3A/LXXAA FoxO1 with the indicated antibody. The cross-linked DNA was amplified by PCR using a set of primers spanning the forkhead binding sites in the Igfbp-1, G6Pase, or Pepck promoter. (C) Hepatic glycogen content. Leprdb/db mice at day 4 after injection with adenovirus encoding LacZ ( $n=3$, white bar), 3A FoxO1 ( $n=3$, gray bar), or 3A/LXXAA FoxO1 ( $n=3$, black bar) were subjected to an overnight fast and killed the next morning for determination of glycogen levels in liver extracts. ${ }^{*} S t a t i s t i c a l l y$ significant difference between $3 \mathrm{~A}$ and $3 \mathrm{~A} / \mathrm{LXXAA}, P<0.02$ by 1 -way ANOVA; ** statistically significant difference between LacZ and $3 A / L X X A A$, $P<0.05$ by 1 -way ANOVA.

8-Br-cAMP, $0.5 \mathrm{mM}$ IBMX, and $1 \mu \mathrm{M}$ dexamethasone in the absence or presence of indicated material was performed and cells were harvested. Luciferase assays were performed according to manufacturer's protocols.

Western blot analysis. We lysed cells in buffer containing $50 \mathrm{mM}$ Tris$\mathrm{HCl}$ (pH 7.6), $250 \mathrm{mM} \mathrm{NaCl}, 1 \% \mathrm{NP}-40,0.5 \%$ deoxycholate, $0.1 \%$ SDS, 50 $\mathrm{mM}$ trichostatin A, $10 \mathrm{mM}$ nicotinamide, and protease inhibitors (Roche Diagnostics). After centrifugation to remove insoluble material, each 30 $\mu \mathrm{g}$ of sample was electrophoresed in $8 \%$ SDS-PAGE, and Western blotting was performed using indicated antibodies. For immunoprecipitation, cell lysates were diluted with buffer containing $50 \mathrm{mM}$ Tris- $\mathrm{HCl}$ ( $\mathrm{pH}$ 7.6), 150 $\mathrm{mM} \mathrm{NaCl}, 0.1 \% \mathrm{NP}-40,10 \%$ glycerol, $5 \mathrm{mM} \mathrm{MgCl}_{2}, 50 \mu \mathrm{M}$ trichostatin A, $10 \mathrm{mM}$ nicotinamide, and protease inhibitors.

Immunofluorescence and immunobistochemistry. Immunofluorescence using SV40-transformed hepatocytes was performed as described previously (52). In the present studies, pCMV5/cMyc/3A FoxO1 or pCMV5/ $\mathrm{cMyc} / 3 \mathrm{~A} / \mathrm{LXXAA}$ FoxO1 was transfected transiently using Lipofectamine (Invitrogen). For immunohistochemistry of liver, we incubated livers overnight in $4 \%$ paraformaldehyde and embedded them in paraffin. We then mounted consecutive $5-\mu \mathrm{m}$ sections on slides. After rehydration and permeabilization, we immunostained sections using rabbit serum against FoxO1 (17). After washing with PBS, we incubated the sections sequentially with biotinylated anti-rabbit IgG reagent (Vector Laboratories) and VECTASTAIN Elite ABC reagent (Vector Laboratories) and visualized with Liquid DAB Substrate Chromogen System (DakoCytomation).

Animal studies. Animals were fed standard chow diet and water ad libitum in sterile cages in a barrier animal facility with a 12/12-hour light/dark cycle. $L e r^{d b / d b}$ mice at the age of 10 weeks were injected through tail veins with an adenoviral vector diluted in PBS buffer at a dose of $4 \times 10^{8} \mathrm{pfu} / \mathrm{g}$ of body weight. Intraperitoneal glucose tolerance test was performed at day 5 after injection. For studies of hepatic gene expression and glycogen measurement, mice were subjected to an overnight fast followed by sacrifice at day 5 after injection. All experimental protocols using mice were approved by the animal ethics committee of Kobe University Graduate School of Medicine.

Analytical procedures and intraperitoneal glucose tolerance test. We measured glucose levels with a Glutest Pro (Sanwa Kagaku Kenkyusho Co.). We carried out all assays in duplicate. Each value represents the means of 2 independent determinations. Hepatic glycogen content at fasting state was measured as described previously (17). For intraperitoneal glucose tolerance test, mice were subjected to an overnight fast followed by intraperitoneal glucose injection $(1.2 \mathrm{~g} / \mathrm{kg})$ as described previously (17).

Tissue RNA isolation and real-time quantitative RT-PCR. Isolation of total RNA from cells was performed using an SV Total RNA Isolation System (Promega) according to manufacturer's protocol. Real-time PCR was also performed as described previously (7).

ChIP assays. Liver sections $(30 \mathrm{mg})$ from $L e p r^{d b / d b}$ mice injected with adenovirus encoding LacZ, 3A FoxO1, or 3A/LXXAA FoxO1 were trypsinized with frequent pipetting and fixed with $1 \%$ formaldehyde for 1 hour at $37^{\circ} \mathrm{C}$. Thereafter, we lysed livers in buffer containing $1 \%$ SDS, $10 \mathrm{mM}$ EDTA, $50 \mathrm{mM}$ Tris- $\mathrm{HCl}$ ( $\mathrm{pH} 8.1$ ), and 0.02 tablets $/ \mathrm{ml}$ of Com-

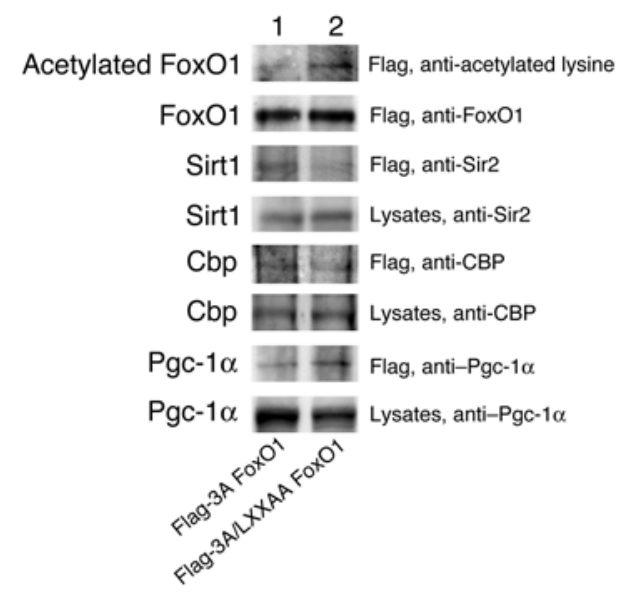

Figure 11

Acetylation of transduced FoxO1 in liver from Lepr ${ }^{d b / d b}$ mice. We sacrificed $L e p r^{d b / d b}$ mice at day 5 after injection of adenovirus encoding 3A (lane 1) or 3A/LXXAA FoxO1 (lane 2) and lysed liver. Liver lysates were immunoprecipitated with anti-Flag monoclonal antibody and Western blotted with the indicated antibodies. 
plete Protease Inhibitor Cocktail (Roche Diagnostics) and sonicated samples using a Bioruptor (Tosho Denki) at maximal power setting with 20 10 -second pulses at $4^{\circ} \mathrm{C}$. Following centrifugation, we diluted the lysates 1:10 in buffer containing 1\% Triton X-100, 2 mM EDTA, $20 \mathrm{mM}$ Tris- $\mathrm{HCl}$ ( $\mathrm{pH} 8.1$ ), and $150 \mathrm{mM} \mathrm{NaCl}$ and precleared them with $30 \mu \mathrm{l}$ of salmon sperm DNA/protein A Sepharose for 2 hours at $4^{\circ} \mathrm{C}$. After removal of the Sepharose beads by centrifugation, we performed immunoprecipitation with anti-Flag monoclonal antibody (M2; Sigma-Aldrich) or an equal amount of normal mouse IgG (Santa Cruz Biotechnology Inc.) and incubated overnight at $4^{\circ} \mathrm{C}$; then, precipitation of the antibody-protein-DNA complexes with salmon sperm DNA/protein A Sepharose for 2 hours at $4{ }^{\circ} \mathrm{C}$ was performed. The precipitates were sequentially washed with buffers containing $0.1 \%$ SDS, $1 \%$ Triton X-100, 2 mM EDTA, and $20 \mathrm{mM}$ Tris- $\mathrm{HCl}$ ( $\mathrm{pH} 8.1$ ) supplemented with either $150 \mathrm{mM}$ (buffer I) or 500 $\mathrm{mM} \mathrm{NaCl}$ (buffer II) prior to a final wash in $250 \mathrm{mM} \mathrm{LiCl}, 1 \% \mathrm{NP}-40,1 \%$ deoxycholate, $1 \mathrm{mM}$ EDTA, and $10 \mathrm{mM}$ Tris- $\mathrm{HCl}$ ( $\mathrm{pH}$ 8.1). The pellets were washed with Tris-EDTA buffer and extracted with $1 \%$ SDS, $10 \mathrm{mM}$ EDTA, and $50 \mathrm{mM}$ Tris- $\mathrm{HCl}$ ( $\mathrm{pH}$ 8.0). After heating at $65^{\circ} \mathrm{C}$ overnight, we digested proteins with proteinase $\mathrm{K}$ and purified DNA as described previously (7). We subjected the samples to PCR using the following primers: for Igfbp-1, 5'-ATCTGGCTAGCAGCTTGCTGA-3' and 5'-CCGTGTGCAGT-
GTTCAATGCT-3'; for G6Pase, 5'-GCCTCTAGCACTCAAGCAGTG-3' and 5'-TGTGCCTTGCCCCTGTTTTATATG-3'; for Pepck, 5'-TCCACCACACACCTAGTGAGG-3' and 5'-AGGGCAGGCCTAGCCGAGACG-3'.

Statistics. We calculated descriptive statistics using ANOVA followed by Fisher's test (Statview; SAS Institute Inc.). $P$ values of less than 0.05 were considered significant.

\section{Acknowledgments}

This work was supported by a grant from the 21st Century Center of Excellence Program "Center of Excellence for Signal Transduction Disease: Diabetes Mellitus as a Model," from the Ministry of Education, Culture, Sports, Science and Technology of Japan.

Received for publication May 2, 2005, and accepted in revised form June 27, 2006.

Address correspondence to: Jun Nakae, Department of Clinical Molecular Medicine, Division of Diabetes, Digestive and Kidney Diseases, Kobe University Graduate School of Medicine, 7-5-1 Kusunoki-cho, Chuo-ku, Kobe 650-0017, Japan. Phone: 81-78-3825374; Fax: 81-78-382-5379; E-mail: nakaej@med.kobe-u.ac.jp.
1. Tran, H., Brunet, A., Griffith, E.C., and Greenberg, M.E. 2003. The many forks in FOXO's road. Sci. STKE. 172:RE5.

2. Accili, D., and Arden, K.C. 2004. FoxOs at the crossroads of cellular metabolism, differentiation, and transformation. Cell. 117:421-426.

3. Kops, G.J., et al. 1999. Direct control of the Forkhead transcription factor AFX by protein kinase B. Nature. 398:630-634.

4. Dijkers, P.F., et al. 2000. Forkhead transcription factor FKHR-L1 modulates cytokine-dependent transcriptional regulation of p27(KIP1). Mol. Cell. Biol. 20:9138-9148.

5. Kops, G.J., et al. 2002. Control of cell cycle exit and entry by protein kinase B-regulated forkhead transcription factors. Mol. Cell. Biol. 22:2025-2036.

6. Medema, R.H., Kops, G.J., Bos, J.L., and Burgering, B.M. 2000. AFX-like forkhead transcription factors mediate cell-cycle regulation by Ras and PKB through p27kip1. Nature. 404:782-787.

7. Nakae, J., et al. 2003. The forkhead transcription factor Foxo1 regulates adipocyte differentiation. Dev. Cell. 4:119-129.

8. Martinez-Gac, L., Marques, M., Garcia, Z., Campanero, M.R., and Carrera, A.C. 2004. Control of cyclin G2 mRNA expression by forkhead transcription factors: novel mechanism for cell cycle control by phosphoinositide 3-kinase and forkhead. Mol. Cell. Biol. 24:2181-2189.

9. Brunet, A., et al. 1999. Akt promotes cell survival by phosphorylating and inhibiting a Forkhead transcription factor. Cell. 96:857-868.

10. Dijkers, P.F., Medema, R.H., Lammers, J.W., Koenderman, L., and Coffer, P.J. 2000. Expression of the pro-apoptotic Bcl-2 family member Bim is regulated by the forkhead transcription factor FKHR-L1. Curr. Biol. 10:1201-1204.

11. Tran, H., et al. 2002. DNA repair pathway stimulated by the forkhead transcription factor FOXO3a through the Gadd45 protein. Science. 296:530-534.

12. Furukawa-Hibi, Y., Yoshida-Araki, K., Ohta, T., Ikeda, K., and Motoyama, N. 2002. FOXO forkhead transcription factors induce $\mathrm{G}(2)-\mathrm{M}$ checkpoint in response to oxidative stress. J. Biol. Chem. 277:26729-26732.

13. Murphy, C.T., et al. 2003. Genes that act downstream of DAF-16 to influence the lifespan of Caenorhabditis elegans. Nature. 424:277-283.

14. Kops, G.J., et al. 2002. Forkhead transcription factor FOXO3a protects quiescent cells from oxidative stress. Nature. 419:316-321.

15. Nemoto, S., and Finkel, T. 2002. Redox regulation of forkhead proteins through a p66shc-dependent signaling pathway. Science. 295:2450-2452.

16. Nakae, J., Kitamura, T., Silver, D.L., and Accili, D. 2001. The forkhead transcription factor Foxo1 (Fkhr) confers insulin sensitivity onto glucose-6phosphatase expression. J. Clin. Invest. 108:1359-1367. doi:10.1172/JCI200112876.

17. Nakae, J., et al. 2002. Regulation of insulin action and pancreatic beta-cell function by mutated alleles of the gene encoding forkhead transcription factor Foxo1. Nat. Genet. 32:245-253.

18. Altomonte, J., et al. 2004. Foxo1 mediates insulin action on apoC-III and triglyceride metabolism. J. Clin. Invest. 114:1493-1503. doi:10.1172/ JCI200419992.

19. Hosaka, T., et al. 2004. Disruption of forkhead transcription factor (FOXO) family members in mice reveals their functional diversification. Proc. Natl. Acad. Sci. U. S. A. 101:2975-2980.

20. Castrillon, D.H., Miao, L., Kollipara, R., Horner, J.W., and DePinho, R.A. 2003. Suppression of ovarian follicle activation in mice by the transcription factor Foxo3a. Science. 301:215-218.

21. Hampsey, M., and Reinberg, D. 1999. RNA polymerase II as a control panel for multiple coactivator complexes. Curr. Opin. Genet. Dev. 9:132-139.

22. Chen, H., Tini, M., and Evans, R.M. 2001. HATs on and beyond chromatin. Curr. Opin. Cell Biol. 13:218-224.

23. McInerney, E.M., et al. 1998. Determinants of coactivator LXXLL motif specificity in nuclear receptor transcriptional activation. Genes Dev. 12:3357-3368

24. Heery, D.M., Kalkhoven, E., Hoare, S., and Parker, M.G. 1997. A signature motif in transcriptional coactivators mediates binding to nuclear receptors. Nature. 387:733-736.

25. Nakae, J., Park, B.C., and Accili, D. 1999. Insulin stimulates phosphorylation of the forkhead transcription factor FKHR on serine 253 through a Wortmannin-sensitive pathway. J. Biol. Chem. 274:15982-15985.

26. Rena, G., Guo, S., Cichy, S.C., Unterman, T.G., and Cohen, P. 1999. Phosphorylation of the transcription factor forkhead family member FKHR by protein kinase B. J. Biol. Chem. 274:17179-17183.

27. Brunet, A., et al. 2004. Stress-dependent regulation of FOXO transcription factors by the SIRT1 deacetylase. Science. 303:2011-2015.

28. Daitoku, H., et al. 2004. Silent information regulator 2 potentiates Foxo1-mediated transcription through its deacetylase activity. Proc. Natl. Acad. Sci. U. S. A. 101:10042-10047.

29. Motta, M.C., et al. 2004. Mammalian SIRT1 represses forkhead transcription factors. Cell. 116:551-563.

30. van der Horst, A., et al. 2004. FOXO4 is acetylated upon peroxide stress and deacetylated by the longevity protein hSir2(SIRT1). J. Biol. Chem. 279:28873-28879.

31. Howitz, K.T., et al. 2003. Small molecule activators of sirtuins extend Saccharomyces cerevisiae lifespan. Nature. 425:191-196.

32. Frescas, D., Valenti, L., and Accili, D. 2005. Nuclear trapping of the forkhead transcription factor FoxO1 via Sirt-dependent deacetylation promotes expression of glucogenetic genes. J. Biol. Chem. 280:20589-20595.

33. Altomonte, J., et al. 2003. Inhibition of Foxo1 function is associated with improved fasting glycemia in diabetic mice. Am. J. Physiol. Endocrinol. Metab. 285:E718-E728.

34. Imai, S., Armstrong, C.M., Kaeberlein, M., and Guarente, L. 2000. Transcriptional silencing and longevity protein Sir2 is an NAD-dependent histone deacetylase. Nature. 403:795-800.

35. Smith, J.S., et al. 2000. A phylogenetically conserved $\mathrm{NAD}+$-dependent protein deacetylase activity in the Sir2 protein family. Proc. Natl. Acad. Sci. U. S. A. 97:6658-6663.

36. Tissenbaum, H.A., and Guarente, L. 2001. Increased dosage of a sir-2 gene extends lifespan in Caenorhabditis elegans. Nature. 410:227-230.

37. Luo, J., et al. 2001. Negative control of p53 by Sir2alpha promotes cell survival under stress. Cell. 107:137-148.

38. Vaziri, H., et al. 2001. hSIR2(SIRT1) functions as an NAD-dependent p53 deacetylase. Cell. 107:149-159.

39. Langley, E., et al. 2002. Human SIR2 deacetylates p53 and antagonizes PML/p53-induced cellular senescence. EMBO J. 21:2383-2396.

40. Cheng, H.L., et al. 2003. Developmental defects and p53 hyperacetylation in Sir2 homolog (SIRT1)-deficient mice. Proc. Natl. Acad. Sci. U. S. A. 100:10794-10799.

41. Fulco, M., et al. 2003. Sir2 regulates skeletal muscle differentiation as a potential sensor of the redox state. Mol. Cell. 12:51-62. 
42. Frye, R.A. 2000. Phylogenetic classification of prokaryotic and eukaryotic Sir2-like proteins. Biochem. Biophys. Res. Commun. 273:793-798.

43. Rodgers, J.T., et al. 2005. Nutrient control of glucose homeostasis through a complex of PGC1alpha and SIRT1. Nature. 434:113-118.

44. Borra, M.T., Smith, B.C., and Denu, J.M. 2005. Mechanism of human SIRT1 activation by resveratrol. J. Biol. Chem. 280:17187-17195.

45. Kaeberlein, M., et al.2005. Substrate-specific activation of sirtuins by resveratrol. J. Biol. Chem. 280:17038-17045.

46. Viswanathan, M., Kim, S.K., Berdichevsky, A., and
Guarente, L. 2005. A role for SIR-2.1 regulation of ER stress response genes in determining C. elegans life span. Dev. Cell. 9:605-615.

47. Gan, L., et al. 2005. FoxO-dependent and -independent mechanisms mediate SirT1 effects on IGFBP-1 gene expression. Biochem. Biophys. Res. Commun. 337:1092-1096.

48. Puigserver, P., et al. 2003. Insulin-regulated hepatic gluconeogenesis through FOXO1-PGC-1alpha interaction. Nature. 423:550-555.

49. van der Heide, L.P., and Smidt, M.P. 2005. Regulation of FoxO activity by $\mathrm{CBP} / \mathrm{p} 300$-mediated acetylation. Trends Biochem. Sci. 30:81-86.
50. Berdichevsky, A., Viswanathan, M., Horvitz, H.R., and Guarente, L. 2006. C. elegans SIR-2.1 interacts with 14-3-3 proteins to activate DAF-16 and extend life span. Cell. 125:1165-1177.

51. Yamagata, K., et al. 2004. Bile acids regulate gluconeogenic gene expression via small heterodimer partner-mediated repression of hepatocyte nuclear factor 4 and Foxo1. J. Biol. Chem. 279:23158-23165. 52. Nakae, J., Barr, V., and Accili, D. 2000. Differential regulation of gene expression by insulin and IGF-1 receptors correlates with phosphorylation of a single amino acid residue in the forkhead transcription factor FKHR. EMBOJ. 19:989-996. 